\title{
Revitalización de la expresión oral quechua como segunda lengua utopía o realidad
}

\author{
Revitalization of Quechua oral expression as a second language utopia or reality
}

Revitalização da expressão oral quíchua como utopia ou realidade de segunda língua

\section{ARTÍCULO DE INVESTIGACIÓN}

\section{Jeannette Marisa Choque Bustinza \\ jmchoque07@gmail.com \\ https://orcid.org/0000-0002-9630-4839}

Universidad Nacional del Altiplano, Puno-Perú

Nelly Olga Zela Payi
nzela@unap.edu.pe

https://orcid.org/0000-0001-9119-1102

Universidad Nacional del Altiplano, Puno-Perú

\author{
Gabriela Cornejo Valdivia \\ gcornejo@unap.edu.pe \\ https://orcid.org/0000-0002-2411-469X
}

Universidad Nacional del Altiplano, Puno-Perú

Martha Ticona Mamani

marthaticona@unap.edu.pe

https://orcid.org/0000-0003-0474-2346

Universidad Nacional del Altiplano, Puno-Perú

Artículo recibido en junio 2021, revisado en julio 2021, arbitrado en agosto 2021 y publicado en septiembre 2021

\section{RESUMEN}

El estudio tuvo como propósito determinar la influencia de los talleres de elaboración de alimentos andinos para la revitalización de la expresión oral del quechua como segunda lengua. Con enfoque cuantitativo de tipo experimental, diseño cuasi experimental, la técnica utilizada fue la observación, y el instrumento la guía de observación, con dos grupos; uno de control y otro experimental, para los resultados se aplicó la $\mathrm{T}$ de student, aceptándose la hipótesis alterna, el experimento se realizó durante tres meses exponiendo al objeto de estudio a una serie de talleres, en la elaboración de alimentos andinos, expresándose en todo momento en lengua quechua dentro del aula y fuera de ella. En conclusión se evidenció que la revitalización de la lengua quechua como L2 es una realidad, obteniéndose cambios positivos en la expresión oral, en su pronunciación, vocabulario y fluidez demostrando una mejora individual en lo mencionado.

Palabras clave: Alimentos; Revitalización; Oralidad; Quechua; Qengua

\section{ABSTRACT}

The aim of the study was to determine the influence of Andean food processing workshops on the revitalization of the oral expression of Quechua as a second language. With a quantitative approach of experimental type, quasi-experimental design, the technique used was observation, and the instrument was the observation guide, with two groups; one control group and the other experimental. For the results the student $\mathrm{T}$ was applied, accepting the alternative hypothesis. The experiment was carried out for three months exposing the object of study to a series of workshops, in the elaboration of Andean foods, expressing itself in all time in Quechua language inside and outside the classroom. In conclusion, it was evident that the revitalization of the Quechua language as L2 is a reality, obtaining positive changes in oral expression, pronunciation, vocabulary and fluency, demonstrating an individual improvement in the above.

Key words: Food; Revitalization; Orality; Quechua; Language

\section{RESUMO}

O objetivo do estudo foi determinar a influência dos workshops de processamento de alimentos andinos para a revitalização da expressão oral de Quechua como segunda língua. Com uma abordagem quantitativa do tipo experimental, desenho quase experimental, a técnica utilizada foi a observação, e o instrumento o guia de observação, com dois grupos; uma de controlo e outra experimental, para os resultados aplicados pelo $\mathrm{T}$ do aluno, aceitando a hipótese alternativa, a experiência foi realizada durante três meses expondo o objeto de estudo a uma série de workshops, na elaboração de alimentos andinos, expressandose sempre em quechua linguagem dentro e fora dela. Em conclusão, ficou demonstrado que a revitalização da língua quechua como L2 é uma realidade, obtendo mudanças positivas na expressão oral, na pronúncia, no vocabulário e na fluência demonstrando uma melhoria individual no que precede.

Palavras-chave: Comida; Revitalização; Oralidade; Quechua; Língua 


\section{INTRODUCCIÓN}

Revitalizar en una segunda lengua originaria se refiere a rescatar la identidad y el desarrollo sociocultural que los pueblos quechuas exigen. (Lovon, 2016). Según se señala que al morir una lengua indígena, muere todo un legado cultural con ella. Muchas lenguas indígenas se encuentran en serio peligro de extinguirse completamente. (Aguirre et al., 2019). Se debe impulsar el acercamiento de los abuelos o sabios de la comunidad a la escuela o viceversa quienes son poseedores de una infinidad de conocimientos ancestrales, los cuales se deben transmitir de generación en generación, resultando una estrategia muy pertinente para fortalecer y revalorar su cultura. De lograrse, la revitalización implicaría reflexionar indirectamente sobre la cultura escolar vigente y la posibilidad de enriquecer la visión de la escuela y de la educación, desde las cosmovisiones y prácticas educativas indígenas propias (Aguirre et al., 2019).

Cuando hay perdida lingüística, es más posible brindar una enseñanza en castellano como la lengua uno (L1), que vendría a ser la L1 de los niños, y luego incluir la lengua quechua que será la lengua dos (L2). (Galdamen y Walqui, 2006).

Para la revitalización de la lengua Quechua es muy imprescindible el papel que juega la educación, y justamente ahí, es donde se debe brindar espacios, para que se siga hablando, reivindicando y fortaleciendo no solo como lengua sino también como cultura, lo cual disminuye de alguna manera la estigmatización de la lengua y la cultura quechua. (Dowman, 2013). La lengua materna o primera lengua (L1) a veces puede llegar a ser la segunda lengua (L2) por la incursión del castellano como lengua de mayor jerarquía Lovon (2016). Para continuar con la práctica de una lengua se debe crear actitudes lingüísticas positivas hacia una determinada lengua en las sociedades de hoy empleándolas en diferentes lugares por ejemplo enseñándolas en las escuelas resultando de vital importancia en el mantenimiento lingüístico de las lenguas quechuas y revitalización. (Aguirre et al., 2019).

La veloz aproximación de centenares de lenguas a la extinción ha imbuido de gran urgencia a este debate, al igual que a las iniciativas a favor de la revitalización lingüística que han surgido a todos los niveles en los últimos años. (Marabini, Mart, Garc, y Humanas, 2020). Una de las mayores riquezas de la humanidad la constituyen sus lenguas, que a su vez son espejo de las distintas culturas que habitan el planeta. Una lengua es una construcción social. (Hern y Farf) La Asamblea General de la ONU aprobó la Declaración sobre los Derechos de los Pueblos Indígenas. En ese documento se menciona que "Los pueblos indígenas tienen derecho a revitalizar, utilizar, fomentar y transmitir a las generaciones futuras sus historias, idiomas, tradiciones orales, filosofía, sistemas de escritura y literaturas. (Garcés, 2020).

Es claro que los procesos de revitalización lingüística y cultural de cualquier lengua, se desarrollan a paso lento y se requiere de mucho esfuerzo, mano de obra capacitada, continuidad, paciencia y recursos para lograr obtener un panorama mucho más clarificador (Itzel, 2017). Cuanto mayor era el riesgo, más se acentuaba la tendencia de la lingüística aplicada a convertirse en forense: documentar las lenguas al borde de su extinción, al mismo tiempo que se acumulaban investigaciones académicas en archivos de casi imposible acceso para los pueblos convertidos en objeto pasivo, condenados a la desaparición. (Ruiz et al., s.f.) Todo esto puede también ser cifrado en términos de estrategias para la sobrevivencia cultural y lingüística(Antonio y Farfán, 2001). Los niños logran en su entorno cotidiano expresarse de 
forma natural, lo cual, le permite satisfacer diversas funciones comunicativas; no obstante, las situaciones escolares, mediante la participación premeditada de los docentes y la intercomunicación con otros niños y niñas conlleva al aprendizaje y desarrollo de nuevas y más complicadas formas lingüísticas y comunicativas, propicios para desempeñarse en nuevas situaciones y contextos. (Galdames et al., 2006). Por otro lado, la comunicación es un proceso que permite a los miembros de una cultura dar a conocer sus ideas, sentimientos, sensaciones acerca del mundo, con un fin determinado (Arevalo, Pardo y Vigil, 2005). La competencia comunicativa aplicada a la enseñanza de una segunda lengua consta de cuatro competencias: gramatical, sociolingüística, discursiva y estratégica. Se basa en el conocimiento que el estudiante tiene sobre la gramática de la segunda lengua. Al que se incorpora el conocimiento del vocabulario, morfología, sintaxis y la pronunciación. (Arevalo et al., 2005).

En elimperioincaicolalengua de mayor dominio $y$ prestigio fue el quechua cambiando radicalmente en la conquista y la colonia convirtiéndose en una lengua de menor prestigio y siendo sometida a la nueva lengua dominante y de prestigio, el castellano, manteniéndose esta situación hasta la actualidad, después que se declaró la independencia de las repúblicas en las que se hablaba el quechua reconocen al castellano como lengua oficial (...) (Zuñiga, 1989). Así mismo los saberes utilizados por sus pobladores desde las antiguas civilizaciones Colla, Inka y Pucara, expresiones culturales, rituales y agrícolas asociadas. (Guardia, 2020). Cuando una lengua ya está en proceso de desaparecer, ciertamente entre los pocos que la siguen todavía manteniendo, hablando, hay cierta vergüenza, por no haberse sumado al abandono generalizado, lo cual implica ser "los más pobres", "los más atrasados", "los más incivilizados" "los más indios". (Chirinos, 1998).
Es así que en la actualidad se puede encontrar niños que ya dejaron de hablar la lengua quechua, tampoco sus padres lo hacen y estetipo de situación es desatendida por la EIB, lo cual merece ser priorizada y estudiada, pues la planificación educativa debe responder a la realidad sociolingüística y educativa que la situación actual amerita. (Lovon, 2016). Para que se dé, un verdadero cambio, primeramente, se debe enfatizar la desigual de la situación lingüística escolar, enmendando este grave error, valorando por igual todas las diferentes lenguas con su variedad y usos lingüísticos (Minedu, 2015). Por lo tanto, el objetivo planteado fue: Determinar la influencia de los talleres de elaboración de alimentos andinos en la pronunciación, vocabulario y fluidez de los niños, planteándose la hipótesis. Los talleres de elaboración de alimentos andinos influyen positivamente para la revitalización de la expresión oral del quechua como L2 en los niños. Los antepasados tuvieron la lengua quechua como primera lengua. Por tal razón, es importante buscar espacios que permitan a los niños y niñas seguir hablando la lengua quechua, que hoy llega a ser la segunda lengua. Es por ello que a través de estos talleres se les brindó espacios para que los niños hablen en quechua como (L2) que consistió en elaborar alimentos andinos con recursos de su zona, donde se expresaban en quechua sin temor ni vergüenza, ni sentirse excluidos, revalorando y fortaleciendo no solamente su lengua, también su cultura, estos talleres sirvieron para que mejoraran su expresión oral de la lengua quechua en cuanto a la pronunciación, vocabulario y fluidez habiendo mejoras.

\section{MÉTODO}

El estudio se realizó en la Institución Educativa Inicial "Milluni" el cual se encuentra ubicado en el distrito de San Antón situado en el noroeste de la Provincia de Azángaro en la zona norte del 
departamento de Puno, Perú con una superficie de 514,84 Km. Los niños y niñas provienen de esa zona, siendo en su mayoría hijos de agricultores, ganaderos y empleados eventuales; los habitantes se dedican a la agricultura y ganadería. Algunos padres son jóvenes, habiendo terminado su educación secundaria, otros solo primaria y otras de más edad no se educaron. Para el grupo control se consideró a la Institución Educativa Inicial $\mathrm{N}^{\circ}$ 835, que se encuentra en la comunidad de Quilca Viluyo a media hora de caminata de la comunidad campesina de Milluni, ubicado en el distrito de San
Antón, provincia de Azángaro departamento de Puno, Perú.

El enfoque de la investigación fue cuantitativo, de tipo experimental, diseño cuasi- experimental con dos grupos; control y experimental. Este tipo de investigación se caracteriza por la manipulación de la variable independiente (causa) con el fin de generar determinados cambios en la variable dependiente (efecto). (Charaja, 2018).

Diagrama del diseño cuasi experimental de dos grupos con pre y pos prueba.
Gc: O1
Ge: $\mathrm{O} 1$

( )

$\mathrm{O} 2$

Se evaluó a los niños y niñas de ambos grupos, control y experimental con el pre test para ver la situación actual en cuanto a su expresión oral, luego se ejecutó el experimento, con el grupo experimental, el experimento implicó realizar 15 talleres con una duración de casi 1 hora con 45 minutos, el taller consistía en la elaboración de alimentos andinos con la participación activa de los niños y niñas comunicándose en todo momento en quechua para revitalizar su segunda lengua, durante tres meses aproximadamente. Al concluir el experimento se aplicó una prueba de salida (pos test) a ambos grupos tanto control como experimental para determinar qué resultados se han obtenido luego de aplicar el experimento. La prueba de hipótesis planteada fue:

H1: Los talleres de elaboración de alimentos andinos influyen positivamente en la revitalización de la expresión oral del quechua como lengua dos.
H0: Los talleres de elaboración de alimentos andinos no influyen para la revitalización de la expresión oral del quechua como Lengua dos.

\section{Nivel de significancia}

$\alpha=0,05$, es decir se trabajó con un margen de error de $5 \%$ y el grado de significancia es de $0.95 \%$, es decir el 95\%.

Estadística de Prueba: la prueba estadística aplicada fue la $\mathrm{T}$ Student porque el número de observaciones es igual o menor a 30 .

La T de Student a esta prueba estadística se utilizó para comparar los resultados antes y después de la aplicación del experimento.

$$
t=\frac{\bar{X}_{1}-\bar{X}_{2}}{\sigma_{p} \sqrt{\frac{1}{N_{1}}+\frac{1}{N_{2}}}}
$$


Donde:

$\mathrm{t}=$ Valor calculado de $\mathrm{t}$ de Student

$\bar{X}_{1}=$ Valor promedio antes

$\bar{X}_{2}=$ Valor promedio después

$\begin{array}{lll}\text { Estadígrafos } & \text { Valores } & \\ \text { Media } & \text { Grupo experimental } & \text { Grupo control } \\ \text { Desviación estándar } & -1,55556 & -0,167 \\ \text { No de discentes } & 0,52705 & 0,408 \\ \text { Valor-p (bilateral) } & 9 & 6 \\ \text { Alfa } & 0,000 & 0,363 \\ \text { Grados de libertad } & 0.05 & 0.05 \\ \text { T Calculada } & 8 & 5 \\ \text { T tabulada } & 8,8545 & -1,000 \\ \end{array}$

\section{b) Regla de decisión}

\section{RESULTADOS Y DISCUSIÓN}

- T calculada > T tabulada, se rechaza la $\mathrm{H}_{\mathrm{o}}$ y se acepta la $\mathrm{H}_{\mathrm{a}}$.

- T calculada < T tabulada, se rechaza la $\mathrm{H}_{\mathrm{a}}$ y se acepta la $\mathrm{H}_{\mathrm{o}}$.

- $\quad$ T Calculada $=8,8545$

- $\quad$ T Tabulada $=1,8595$

El valor calculado para el grupo experimental es 8,8545 por lo tanto se contrasta contra el valor tabular que para 9 grados de libertad es de 1,8595.

\section{Interpretación y decisión de la aplicación del diseño estadístico}

Como la $\mathrm{T}$ calculada para el grupo experimental (8.8545) es mayor a la $\mathrm{T}$ tabulada (1.8595) es altamente positiva, por lo que se rechaza la hipótesis nula y se acepta la hipótesis alterna, lo que quiere decir que los talleres de elaboración de alimentos andinos influyen positivamente para la revitalización de la expresión oral del quechua.

Para la obtención de los resultados se aplicó la guía de observación, con una duración de tres meses exponiendo al objeto de estudio a una serie de talleres en la elaboración de alimentos andinos, expresándose en todo momento en lengua quechua dentro del aula y fuera de ella.

De acuerdo a la Tabla 1, aplicada la prueba de entrada (Pre test) del grupo experimental y de control. Se observó que en la escala logro previsto, tanto en el grupo experimental como de control no se ubica ningún niño (0\%). En la escala en proceso, en el grupo experimental se ubican dos niños (22\%) y en el grupo control se ubica un niño (17\%). En la escala en inicio, en el grupo experimental existen siete niños (78\%), mientras que en el grupo control se ubican cinco niños (83\%) podemos deducir que los niños se comunican más en castellano y muy pocas veces hablan el quechua. También se muestra en los resultados que en la prueba de salida (Post test) los niños y niñas del grupo experimental y de control. Se observa que en la escala logro previsto, 
en el grupo experimental se ubican siete niños (78\%) y en el grupo control no se ubica ningún estudiante (0\%). En la escala en proceso, en el grupo experimental se ubican dos niños (22\%) y en el grupo control se ubican dos niños (33\%). en inicio, en el grupo experimental no existe ningún niño
(0\%), mientras que en el grupo de control se ubican cuatro niños (67\%). Estos resultados significan que en la mayoría de estudiantes del grupo experimental (78\%), después de aplicar los talleres se ubicaron en logro previsto, de esa manera el experimento aplicado dio resultados positivos.

Tabla 1. Revitalización de la expresión oral quechua como L2.

\begin{tabular}{|c|c|c|c|c|c|c|c|c|}
\hline \multirow[b]{3}{*}{ Escala } & \multicolumn{4}{|c|}{ Experimental } & \multicolumn{4}{|c|}{ Control } \\
\hline & \multicolumn{2}{|c|}{ Pre-test } & \multicolumn{2}{|c|}{ Post-test } & \multicolumn{2}{|c|}{ Pre-test } & \multicolumn{2}{|c|}{ Post-test } \\
\hline & $\mathbf{F i}$ & hi\% & fi & hi\% & $\mathbf{F i}$ & hi\% & fi & hi\% \\
\hline Logro previsto & 0 & $0 \%$ & 7 & $78 \%$ & 0 & $0 \%$ & 0 & $0 \%$ \\
\hline En proceso & 2 & $22 \%$ & 2 & $22 \%$ & 1 & $17 \%$ & 2 & $33 \%$ \\
\hline En inicio & 7 & $78 \%$ & 0 & $0 \%$ & 5 & $83 \%$ & 4 & $67 \%$ \\
\hline Total & 9 & $100 \%$ & 9 & $100 \%$ & 6 & $100 \%$ & 6 & $100 \%$ \\
\hline
\end{tabular}

Fuente: Guía de observación

De acuerdo a la Tabla 2, se describe los resultados de la prueba de entrada (Pre test) de los niños del grupo experimental y de control en cuanto a pronunciación. En la escala logro previsto, tanto en el grupo experimental como de control no se ubica ningún niño (0\%). En la escala en proceso, en el grupo experimental se ubican dos niños (22\%) y en el grupo control se ubican dos niños (33\%). En la escala en inicio, en el grupo experimental siete niños (78\%), mientras que en el grupo de control cuatro niños (67\%). También se muestra en los resultados que en la prueba de salida (Pos test) de los niños del grupo experimental y de control en cuanto a pronunciación se observa que en la escala logro previsto del grupo experimental se ubican seis niños (67\%) y en el grupo control un niño (17\%). En la escala en proceso, en el grupo experimental se ubican dos niños (22\%) y en el grupo control se ubica un niño (17\%) en inicio, en el grupo experimental existe un niño (11\%), mientras que en el grupo de control se ubican cuatro niños (66\%). Estos resultados significan que en la mayoría del grupo experimental (67\%), se ubicaron en logro previsto, de esa manera el experimento aplicado dio resultados positivos.

Tabla 2. Revitalización de la expresión oral del quechua como L2 en la dimensión Pronunciación.

\begin{tabular}{|c|c|c|c|c|c|c|c|c|}
\hline \multirow{3}{*}{$\begin{array}{l}\text { Pronunciación } \\
\text { Escala }\end{array}$} & \multicolumn{4}{|c|}{ Experimental } & \multicolumn{4}{|c|}{ Control } \\
\hline & \multicolumn{2}{|c|}{ Pre-test } & \multicolumn{2}{|c|}{ Post-test } & \multicolumn{2}{|c|}{ Pre-test } & \multicolumn{2}{|c|}{ Post-test } \\
\hline & $\mathbf{F i}$ & hi\% & fi & hi\% & $\mathbf{F i}$ & hi\% & fi & hi\% \\
\hline Logro previsto & 0 & $0 \%$ & 6 & $67 \%$ & 0 & $0 \%$ & 1 & $17 \%$ \\
\hline En proceso & 2 & $22 \%$ & 2 & $22 \%$ & 2 & $33 \%$ & 1 & $17 \%$ \\
\hline En inicio & 7 & $78 \%$ & 1 & $11 \%$ & 4 & $67 \%$ & 4 & $66 \%$ \\
\hline Total & 9 & $100 \%$ & 9 & $100 \%$ & 6 & $100 \%$ & 6 & $100 \%$ \\
\hline
\end{tabular}

Fuente: Guía de observación 
De acuerdo a la Tabla 3, los resultados de la prueba de entrada (Pre test) de los niños del grupo experimental y de control en cuanto a vocabulario. En la escala logro previsto, en el grupo experimental se ubica un niño que representa el (11\%) y en el grupo control no se ubica ningún niño (0\%). En la escala en inicio, en el grupo experimental se ubica un niño (11\%), en el grupo control se ubica un niño (17\%). En la escala en inicio, en el grupo experimental existen siete niños (78\%), mientras que en el grupo de control se ubican cinco niños (83\%). También se muestra en los resultados de la prueba de salida (Pos test) de los estudiantes del grupo experimental y de control en cuanto a vocabulario se observó que en la escala logro previsto, en el grupo experimental se ubican siete niños $(78 \%)$ y en el grupo control no se ubica ningún estudiante (0\%). En la escala en proceso, en el grupo experimental se ubican dos niños $(22 \%)$ y en el grupo control se ubica dos niños (33\%). En la escala en inicio, en el grupo experimental no existe ningún estudiante (0\%), mientras que en el grupo de control se ubican cuatro niños (67\%). Estos resultados significan que en la mayoría de niños del grupo experimental (78\%), se encuentran en logro previsto, de esa manera el experimento aplicado dio resultados positivos.

Tabla 3. Revitalización de la expresión oral del quechua como L2 en la dimensión Vocabulario.

\begin{tabular}{|c|c|c|c|c|c|c|c|c|}
\hline \multirow{3}{*}{$\begin{array}{l}\text { Vocabulario } \\
\text { Escala }\end{array}$} & \multicolumn{4}{|c|}{ Experimental } & \multicolumn{4}{|c|}{ Control } \\
\hline & \multicolumn{2}{|c|}{ Pre-test } & \multicolumn{2}{|c|}{ Post-test } & \multicolumn{2}{|c|}{ Pre-test } & \multicolumn{2}{|c|}{ Post-test } \\
\hline & $\mathbf{F i}$ & hi\% & fi & hi\% & $\mathbf{F i}$ & hi\% & fi & hi\% \\
\hline Logro previsto & 1 & $11 \%$ & 7 & $78 \%$ & 0 & $0 \%$ & 0 & $0 \%$ \\
\hline En proceso & 1 & $11 \%$ & 2 & $22 \%$ & 1 & $17 \%$ & 2 & $33 \%$ \\
\hline En inicio & 7 & $78 \%$ & 0 & $0 \%$ & 5 & $83 \%$ & 4 & $67 \%$ \\
\hline Total & 9 & $100 \%$ & 9 & $100 \%$ & 6 & $100 \%$ & 6 & $100 \%$ \\
\hline
\end{tabular}

Fuente: Guía de observación

En la Tabla 4, los resultados de la prueba de entrada (Pres test) de los niños del grupo experimental y de control en cuanto a fluidez. En la escala de logro previsto, tanto en el grupo experimental como en el grupo control no se ubica ningún niño (0\%). En la escala en proceso, en el grupo experimental se ubica un niño (11\%) y en el grupo control se ubica un niño (17\%). En la escala en inicio, en el grupo experimental existen ocho niños (89\%), mientras que en el grupo control se ubican cinco niños (83\%). También se muestra en los resultados de la prueba de salida (Pos test) de los niños del grupo experimental y de control en cuanto a fluidez se observó en la escala logro previsto, en el grupo experimental se ubican seis niños (67\%) $y$ en el grupo control se ubica un niño (17\%). En la escala en proceso, en el grupo experimental se ubican tres niños (33\%) y en el grupo control se ubica un niño (17\%). En la escala en inicio, en el grupo experimental no existe ningún estudiante (0\%), mientras que en el grupo de control se ubican cuatro niños (66\%). Estos resultados significan que en la mayoría de los niños del grupo experimental (67\%), se ubicaron en logro previsto, de esa manera el experimento aplicado dio resultados positivos. Para ello, se desarrolló talleres para que no haya una perdida lingüística, y sea posible brindar una enseñanza en castellano como la lengua uno (L1), que vendría a ser la L1 de los niños, y luego incluir la lengua quechua que será la lengua dos (L2). (Galdames y Walqui, 2006). 
Tabla 4. Revitalización de la expresión oral del quechua como L2 en la dimensión Fluidez.

\begin{tabular}{lcccccccc}
\hline \multicolumn{1}{c}{ Fluidez } & \multicolumn{4}{c}{ Experimental } & \multicolumn{3}{c}{ Control } \\
& \multicolumn{2}{c}{ Pre-test } & \multicolumn{2}{c}{ Post-test } & Pre-test & Post-test \\
\hline Escala & Fi & $\mathbf{h i} \%$ & $\mathbf{f i}$ & $\mathbf{h i} \%$ & $\mathbf{F i}$ & $\mathbf{h i} \%$ & $\mathbf{f i}$ & $\mathbf{h i} \%$ \\
Logro previsto & 0 & $0 \%$ & 6 & $67 \%$ & 0 & $0 \%$ & 1 & $17 \%$ \\
En proceso & 1 & $11 \%$ & 3 & $33 \%$ & 1 & $17 \%$ & 1 & $17 \%$ \\
En inicio & 8 & $89 \%$ & 0 & $0 \%$ & 5 & $83 \%$ & 4 & $66 \%$ \\
Total & $\mathbf{9}$ & $\mathbf{1 0 0} \%$ & $\mathbf{9}$ & $\mathbf{1 0 0} \%$ & $\mathbf{6}$ & $\mathbf{1 0 0} \%$ & $\mathbf{6}$ & $\mathbf{1 0 0} \%$ \\
\hline
\end{tabular}

Fuente: Guía de observación

\section{Discusión}

Se confirma en la Tabla 1, que para revitalizar una lengua se debe crear actitudes lingüísticas positivas hacia una determinada lengua en las sociedades de hoy empleándolas en diferentes lugares por ejemplo enseñándolas en las escuelas resultando de vital importancia en el mantenimiento lingüístico de las lenguas quechuas y revitalización. (Aguirre et al., 2019). La veloz aproximación de centenares de lenguas a la extinción ha imbuido de gran urgencia a este debate, al igual que a las iniciativas a favor de la revitalización lingüística que han surgido a todos los niveles en los últimos años. (Marabini, Mart, Garc, y Humanas, 2020). Estos resultados confirman que la utilización de la lengua quechua se ve limitado a espacios extracurriculares quedando esta lengua subsumida culturalmente, considerando que un buen porcentaje de estudiantes son monolingües españoles también confirman que los padres de familia, así sean mestizos o indígenas no desean que a sus niños se les enseñe en quechua. Toalombo (2015). En los resultados obtenidos de Sánchez (2014), confirman que la aplicación de estrategias comunicativas demostró una contribución significativa en la revitalización oral del quechua, permitiendo revitalizar el idioma quechua aprendieron el manejo de nuevas palabras manejo oral y fluidez identificándose con su cultura. Con la planificación de los talleres se pudo revitalizar la práctica de la expresión oral en el idioma quechua, las estrategias se pueden utilizar para la práctica de la oralidad y así ayudar a evitar la extinción de una de las lenguas originarias.

En la Tabla 2, se observa que hay cierta vergüenza, por no haberse sumado al abandono generalizado, lo cual implica ser "los mas pobres", "los mas atrasados", "los mas incivilizados" "los mas indios". Los que hablan el quechua(Chirinos, 1998). Revitalizar en una segunda lengua originaria se refiere a rescatar la identidad y el desarrollo sociocultural que los pueblos quechuas exigen. (Lovon, 2016). Según se señala que al morir una lengua indígena, muere todo un legado cultural con ella. Muchas lenguas indígenas se encuentran en serio peligro de extinguirse completamente. (Aguirre, Yon, Yon y Reategui del Aguila, 2019). Por ello, estos talleres fueron de vital importancia para que no desaparezca la lengua quechua.

En la Tabla 3, se confirmó que "Los pueblos indígenas tienen derecho a revitalizar, utilizar, fomentar y transmitir a las generaciones futuras sus historias, idiomas, tradiciones orales, filosofía, sistemas de escritura y literaturas. (Garcés, 2020). Es claro que los procesos de revitalización lingüística y cultural de cualquier lengua, se desarrollan a paso lento y se requiere de mucho esfuerzo, mano de 
obra capacitada, continuidad, paciencia y recursos para lograr obtener un panorama mucho más clarificador (Itzel, 2017). Lo cual indica con los talleres se pudo reivindicar la práctica de la oralidad en L2. Los resultados permiten deducir que para enseñar una segunda lengua se debe planificar actividades significativas que sean de interés para el niño.

En la Tabla 4 se devela que para la revitalización de la lengua Quechua es imprescindible el papel que juega la educación, y justamente ahí, es donde se debe brindar espacios, para que se siga hablando, reivindicando y fortaleciendo no solo como lengua sino también como cultura, lo cual disminuye de alguna manera la estigmatización de la lengua y la cultura quechua. (Dowman, 2013). Efectivamente según estos resultados para enseñar una lengua es muy importante planificar actividades vivenciales que sean muy significativos para que el niño aprenda una segunda lengua en la que el niño y niña pueda expresarse en quechua.

\section{CONCLUSIONES}

Los talleres de elaboración de alimentos andinos influyeron positivamente en la revitalización de la expresión oral del quechua como lengua dos (L2). Los niños alcanzaron la categoría de logro previsto y en proceso, lo que significa que lograron expresarse en la lengua quechua mejorando su pronunciación, incrementado su vocabulario $\mathrm{y}$ mejorando su fluidez, rechazándose la hipótesis nula y aceptándose la hipótesis alterna, donde se muestra que existen diferencias estadísticas significativas entre los puntajes obtenidos del grupo experimental y el grupo control.

Los talleres de elaboración de alimentos andinos influyen positivamente en la mejora de la pronunciación de la lengua quechua mostrando mejorías sustanciales, un buen porcentaje se encuentran en la categoría de logro previsto y en proceso, puesto que lograron pronunciar las palabras en quechua de forma clara.

La aplicación de talleres de elaboración de alimentos andinos influyó positivamente en el incremento del vocabulario quechua, un buen porcentaje se encuentra en la categoría de logro previsto y en proceso, lo que evidencia que lograron incrementar palabras nuevas que incorporaron en la comunicación de su vida cotidiana.

El taller de elaboración de alimentos andinos influye positivamente en la fluidez, algunos niños expresan sus ideas con más facilidad que al inicio, encontrándose un buen porcentaje en la categoría logro previsto y en proceso, la fluidez se logró con la práctica oral en los talleres y de la comunicación diaria entre el adulto.

\section{REFERENCIAS}

Aguirre, B. N. (2019). Tratamiento de la Lengua originaria II. Perú: Waras. Lima: PUCP

Antonio, J., y Farfán, F. (2001). El empoderamiento de las lenguas amenazadas: ilustraciones mexicanas

Arevalo, I. P. (2005). Enseñanza del Castellano como Segunda Lengua en las Escuelas EBI del Perú

Chirinos, A. (1998). Las Lenguas Indigenas Peruanas mas alla del 2000. Revista Andina, 453-479

Dowman, S. (2013). El Futuro del Quechua en el Peru ¿se puede Revitalizar? La BloGoteca De Babel, 4(Art. 4)

Galdames, V., y Walqui, A. y. (2006). Enseñanza de Lengua Indigena como Lengua Materna. Mexico: PROEIB Andes

Garcés, F. (2020). La revitalización de las lenguas indígenas. Voces e imágenes de las lenguas en Peligro de Extincion. Quito-Ecuador: Abya- Yala

Guardia, S. (2020). Gastronomíaperuana Patrimonio cultural de la humanidad. Huamanga Ayacucho 2018. Universidad Peruana Union, Huamanga Ayacucho 
Hern, L. C., y Farf, A. F. (s.f.). Guía de revitalización lingüística: para una gestión formada $e$ informada

Itzel, V. (2017). revitalización lingüística del hñähñu (otomí) del Valle del Mezquital, Hidalgo : de actores , discursos y prácticas, 133(4), 10641090

Itzel, V. (2017). Concepto de fluidez en la expresion oral. Obtenido de https://cvc.cervantes.es/ Ensenanza/biblioteca_ele/publicaciones_ centros/PDF/rio_2008/37_horche-marco.pdf

Lovon, C. M. (2016). Enseñanza de la Lengua Vernacula: Como Segunda Lengua: Lenguas Indigenas Peruanas en estado L2. Escritura $y$ Pensamiento, 19, 185-210
Marabini, B., Mart, S., Garc, L. R., y Humanas, C. (2020). Nuevas herramientas para la revitalización lingüística: análisis comparativo de los procesos de revitalización del hebreo y las lenguas indígenas australianas

Ministerio de Educacion (MINEDU). (2015). Rutas del aprendizaje ¿Qué y cómo aprenden nuestros niños? II ciclo area curricular Comunicacion. Lima: Metrocolor S.A

Ruiz, O. P., Alegría, C., Ponce, D., Fernández, M., Leyva, J. G., Pablo, J., Martínez, J. J. (s.f.). Del extractivismo cultural y linguistico a la revitalización de las lenguas (pp. 369-389)

Zuñiga Castillo, M. (1989). Educación Bilingue. Santiago de Chile: S.R.V. Impresos OREALC 